ARTICLE

https://doi.org/10.1038/s41467-019-10256-3

\title{
Gate tunable giant anisotropic resistance in ultra-thin GaTe
}

Hanwen Wang 1,2,13, Mao-Lin Chen 1,2,13, Mengjian Zhu3,13, Yaning Wang 1,2,13, Baojuan Dong 1,2, Xingdan Sun ${ }^{1,2}$, Xiaorong Zhang ${ }^{4,5}$, Shimin Cao ${ }^{6,7}$, Xiaoxi Li1,2, Jianqi Huang ${ }^{1,2}$, Lei Zhang ${ }^{1,2}$, Weilai Liu ${ }^{1,2}$, Dongming Sun (1) 1,2, Yu Ye (i) 7,8, Kepeng Song9 ${ }^{9}$ Jianjian Wang ${ }^{10}$, Yu Han (1) 9, Teng Yang (1) 1,2, Huaihong Guo ${ }^{11}$, Chengbing Qin (1) 4,5, Liantuan Xiao ${ }^{4,5}$, Jing Zhang ${ }^{5,12}$, Jianhao Chen (10 6,7, Zheng Han (1) 1,2,5 \& Zhidong Zhang ${ }^{1,2}$

Anisotropy in crystals arises from different lattice periodicity along different crystallographic directions, and is usually more pronounced in two dimensional (2D) materials. Indeed, in the emerging 2D materials, electrical anisotropy has been one of the recent research focuses. However, key understandings of the in-plane anisotropic resistance in low-symmetry 2D materials, as well as demonstrations of model devices taking advantage of it, have proven difficult. Here, we show that, in few-layered semiconducting GaTe, electrical conductivity anisotropy between $\mathbf{x}$ and $\mathbf{y}$ directions of the 2D crystal can be gate tuned from several fold to over $10^{3}$. This effect is further demonstrated to yield an anisotropic non-volatile memory behavior in ultra-thin GaTe, when equipped with an architecture of van der Waals floating gate. Our findings of gate-tunable giant anisotropic resistance effect pave the way for potential applications in nanoelectronics such as multifunctional directional memories in the 2D limit.

\footnotetext{
${ }^{1}$ Shenyang National Laboratory for Materials Science, Institute of Metal Research, Chinese Academy of Sciences, Shenyang 110016, China. ${ }^{2}$ School of Material Science and Engineering, University of Science and Technology of China, Anhui 230026, China. ${ }^{3}$ College of Advanced Interdisciplinary Studies, National University of Defense Technology, Changsha 410073, China. ${ }^{4}$ State Key Laboratory of Quantum Optics and Quantum Optics Devices, Institute of Laser Spectroscopy, Shanxi University, Taiyuan 030006, China. ${ }^{5}$ Collaborative Innovation Center of Extreme Optics, Shanxi University, Taiyuan 030006, China. ${ }^{6}$ International Center for Quantum Materials, School of Physics, Peking University, Beijing 100871, China. ${ }^{7}$ Collaborative Innovation Center of Quantum Matter, Beijing 100871, China. ${ }^{8}$ State Key Lab for Mesoscopic Physics and School of Physics, Peking University, Beijing, China. ${ }^{9}$ Advanced Membranes and Porous Materials Center, Physical Science and Engineering Division, King Abdullah University of Science and Technology, Thuwal 23966-6900, Saudi Arabia. ${ }^{10}$ Multi-scale Porous Materials Center, Institute of Advanced Interdisciplinary Studies, Chongqing University, Chongqing 400044, China. ${ }^{11}$ College of Sciences, Liaoning Shihua University, Fushun 113001, China. ${ }^{12}$ State Key Laboratory of Quantum Optics and Quantum Optics Devices, Institute of Opto-Electronics, Shanxi University, Taiyuan 030006, China. ${ }^{13}$ These authors contributed equally: Hanwen Wang, Mao-Lin Chen, Mengjian Zhu, and Yaning Wang. Correspondence and requests for materials should be addressed to T.Y. (email: Yangteng@imr.ac.cn) or to C.Q. (email: Chbqin@sxu.edu.cn) or to J.C. (email: jhChen@pku.edu.cn) or to Z.H. (email: vitto.han@gmail.com)
} 
$\mathrm{t}$ is known that when electrically measuring a bulk material, the resulted conductivity may manifest strong directional dependencies ${ }^{1,2}$. Discrepancy of conductivity along different in-plane directions in layered bulk crystals can sometimes be as large as a few hundreds, which, however, often requires a certain conditions such as the presence of large external magnetic field ${ }^{3}$. Recently, low-symmetry $2 \mathrm{D}$ materials have attracted significant attentions because of the potential applications of in-plane anisotropic nanoelectronics ${ }^{4-11}$. For example, ultra-thin black phosphorous flake showed an in-plane anisotropic conductance reaching a ratio $\sigma_{\mathrm{a}} / \sigma_{\mathrm{b}}$ of $\sim 1.5$, which in principle can be a direction-sensitive sensor ${ }^{4}$. $\operatorname{ReS}_{2}$ was reported to be another candidate for anisotropic $2 \mathrm{D}$ field effect transistor, which exhibited a $\sigma_{\mathrm{a}}$ of almost 10 times the value of $\sigma_{\mathrm{b}}{ }^{9}$. Recent studies on $\mathrm{GeP}$ and GeAs flakes also showed anisotropic behavior with anisotropic factors of $1.5 \sim 2$ for their conductance ${ }^{10,11}$.
To date, in-plane anisotropic factor $\Gamma_{\mathrm{a}}$ of electrical conductance in $2 \mathrm{D}$ materials is yet limited within one order of magnitude under ambient condition. It thus hinders future applications owing to a weak effective conductance difference between directions, and a larger anisotropic factor is highly desired. In this work, we show an observation of giant anisotropic resistance (GAR) behavior in few-layered p-type semiconducting GaTe. Among devices measured, the electrical conductivity ratio along $x$ and $y$ ( $x$ and $y$ are defined in Fig. 1) directions of ultra-thin GaTe reaches an order of $10^{3}$ at gate voltages close to the valence-band maximum (VBM), which can be further gate tuned down to $<10$, upon hole doping. We show detailed analysis and physical understandings on the GAR effect. Based on this, floating gate anisotropic memory with directional multi-level outputs are demonstrated. Moreover, when measuring along fixed direction, the few-layered GaTe floating gate memory (FGM) exhibits On/Off ratio of $10^{7}$ and retention time of $10^{5} \mathrm{~s}$, which is by far the

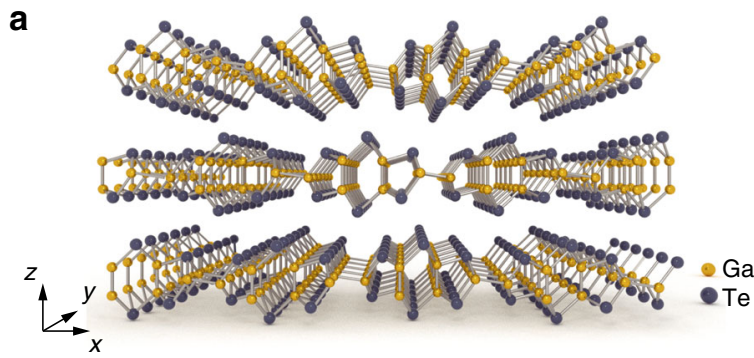

b

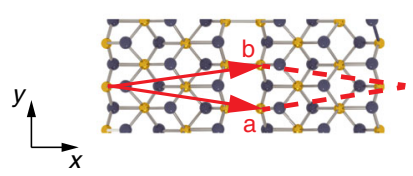

C

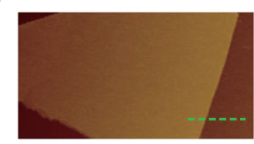

d

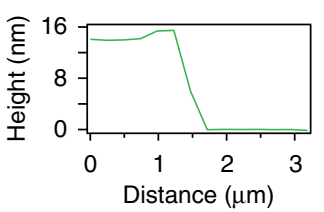

h

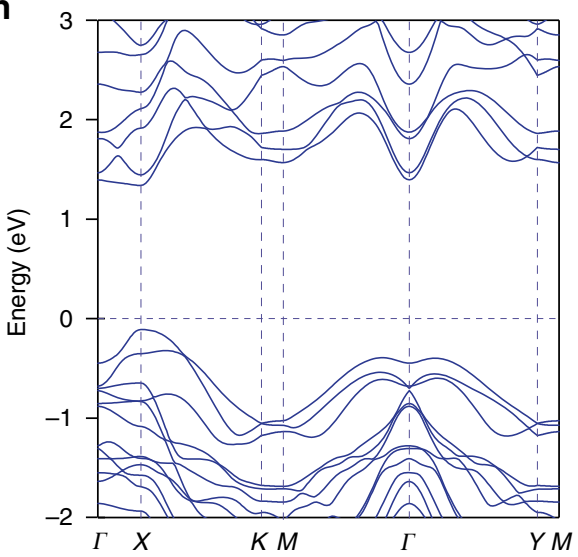

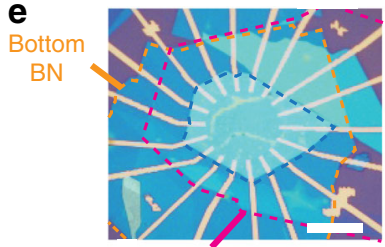

Top BN

$\mathbf{f}$

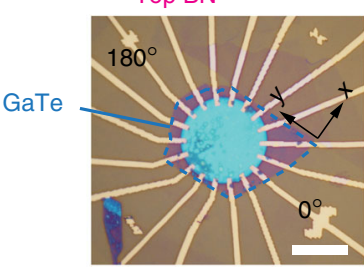

g

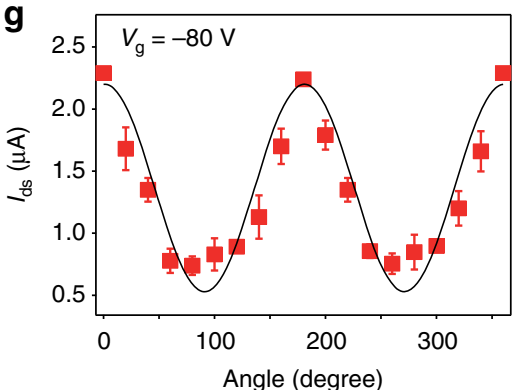

i

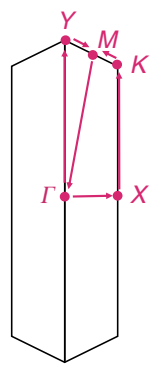

j

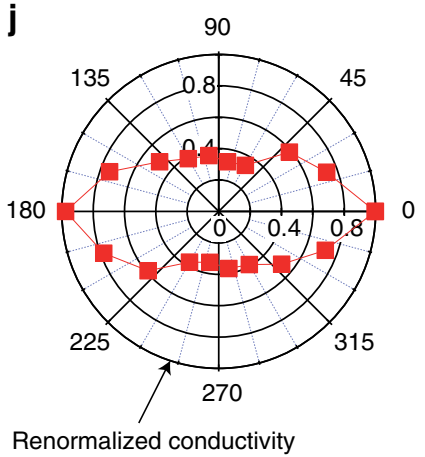

Fig. 1 Characterizations of ultra-thin GaTe. a Schematics of GaTe crystal structure, with its in-plane unit cell illustrated in b. c AFM image of a typical GaTe flake of $~ 14 \mathrm{~nm}$ in thickness, with its height profile along the green dashed line plotted in $\mathbf{d}$. e Optical image of a typical device (sample S1) made of 14 nm GaTe flake encapsulated in h-BN. Electrodes are patterned with an angle interval of 20 degree. $\mathbf{f}$ Same device in e but patterned into a circular shape. Scale bars in $\mathbf{e}$ and $\mathbf{f}$ are $10 \mu \mathrm{m}$. $\mathbf{g}$ Source-drain current at $V_{\mathrm{ds}}=2 \mathrm{~V}$ as a function of angle, with $V_{\mathrm{g}}=-80 \mathrm{~V}$. The black solid line is an ellipsoidal fit. Error bars in $\mathbf{g}$ are defined as the deviations between experimental data and the fitted line. $\mathbf{h}$ Electronic band structure of monolayer GaTe, with the first Brillouin zone plotted in $\mathbf{i}$. $\mathbf{j}$ Same data in $\mathbf{g}$ plotted after re-normalization in a polar graph 
best performance among FGMs made of 2D materials. Our findings open new possibilities toward next generation nanoelectronics, such as artificial neuron network based on anisotropic memory arrays.

\section{Results}

Characterizations of ultra-thin GaTe. Bulk GaTe single crystals were prepared via flux method and were confirmed via x-ray diffraction (see Supplementary Fig. 1). We mechanically exfoliated the bulk and deposited ultra-thin GaTe flakes onto $285 \mathrm{~nm}$ silicon oxide grown on heavily doped silicon wafers for optical and electrical characterizations. It is known that GaTe has a layered structure with lattice symmetry of group $C_{2 h}$ (Fig. 1a), similar to that of $1 \mathrm{~T}^{\prime}-\mathrm{MoTe}_{2}$. The unit cell for a single layer GaTe is indicated in Fig. 1b. Monoclinic GaTe crystal is of very low symmetry, providing an ideal platform to study anisotropy ${ }^{12}$. It is also known as a typical p-type semiconductor with a direct band gap of $\sim 1.65 \mathrm{eV}^{13,14}$, which has many potential applications, such as photodetectors ${ }^{15}$, radiation detectors ${ }^{16}$, as well as thermophotovoltaic devices ${ }^{17}$. However, GaTe in air was reported to be easily oxidized ${ }^{18}$, leading to band gap restructuring in air, at the same time, the photoluminescence (PL) signal decreases and extra Raman peaks associated to oxides become more pronounced. Atomic Force Microscope (AFM) scan of a typical GaTe flake (14 nm in thickness) is shown in Fig. 1c, d. First, we characterized electronic transport of those flakes in air (Supplementary Figs. 2-6). However, without protection from air, the source-drain currents $I_{\mathrm{ds}}$ of devices made by GaTe flakes are rather low, with maximum current of a few nano amperes, in agreement with other reported results ${ }^{19}$. It is found that, without thermal annealing, few-layered GaTe devices are barely conducting, as shown in Supplementary Fig. 2. In the following, we will focus on the devices made by ultra-thin GaTe flakes encapsulated by hexagonal boron nitride (h-BN) in a glove box, in order to diminish damages from air. Detailed analysis of the effect of h-BN protection can be found in Supplementary Figs. 7-11.

Polarized Raman can be used to determine crystal orientation in $2 \mathrm{D}$ flakes ${ }^{10,12}$, and also to deliver information of phonon modes that are related to the lattice symmetry. Supplementary Fig. 12 shows Raman spectra of a typical GaTe flake, with the angle-dependent intensity at Raman shift of $271.1 \mathrm{~cm}^{-1}$ plotted in a polar figure overlaid on the optical image shown in the inset. A twofold symmetry of the polar figure can be seen, with one of the polarized axis parallel to the exfoliated straight edge of the GaTe itself. Such a Raman-active mode is a $A_{\mathrm{g}}$ mode in $C_{2 \mathrm{~h}}$ point group. From Raman tensor analysis, the direction of maximal Raman intensity of such $A_{\mathrm{g}}$ mode corresponds to the lattice direction with short lattice constant, defined as $y$ direction in Fig. 1a, with $x$ direction perpendicular to it. Raman mode at $164.6 \mathrm{~cm}^{-1}$ is another good reference for determining the crystalline direction (see Supplementary Fig. 13) ${ }^{12}$. Such a mode has a fourfold symmetry ( $B_{\mathrm{g}}$ mode) and therefore four-lope angular dependence, with each lope 45 degrees away from the polarization axis of the mode at $271.1 \mathrm{~cm}^{-1}$. By comparing Raman anisotropy with the anisotropy of electrical conductivity (shown in the next section), it is known that $y$ axis of GaTe layer corresponds to a maximum conductivity (Supplementary Figs. 12-13). By this means, one can pattern electrodes for electrical measurements just with the zero angle defined along with such straight edges ( $y$ direction).

As shown in Fig. 1e, nine pairs of electrodes $\left(20^{\circ}\right.$ angle between each two electrodes) were deposited onto the h-BN/GaTe/h-BN stack sample S1 (see Methods), which is further patterned into a circular shape in Fig. 1f. As a result, the source-drain current
$I_{\mathrm{ds}}$ along each pair of electrodes at gate voltage $V_{\mathrm{g}}=-80 \mathrm{~V}$ follows an ellipsoidal dependence of the testing angle, shown in Fig. 1g. It is rather straight forward that the maximum $I_{\mathrm{ds}}$ flows in $0^{\circ}$, which is parallel to one of the straight edges, i.e., the $y$ axis, as marked in Fig. 1f. Such twofold ellipsoidal oscillation of inplane anisotropic conductivity is also seen in a number of other 2D materials ${ }^{4,5,9-11,20}$.

To unveil the origin of in-plane electrical conductivity anisotropy found in ultra-thin GaTe, we performed first-principles electronic structure and non-equilibrium Green's functional quantum transport calculations with a simplified model on GaTe monolayer. Electronic band structure along high-symmetry lines is shown in Fig. 1h, with the first Brillouin zone and high-symmetry points defined in Fig. 1i. A direct band gap of $1.5 \mathrm{eV}$ locates at the $X$ point, in agreement with PL measurement in Supplementary Fig. 14. Thanks to the direct band gap, outstanding photo responses have been reported in GaTe devices ${ }^{15,21}$. What's very interesting is the strongly anisotropic band dispersion obviously seen along two perpendicular directions $(\Gamma-X$ and $X-K)$. At the VBM, band dispersion along $\Gamma-X$ is much bigger than along $X-K$, which gives rise to strong anisotropic effective mass $m^{*}$ at VBM, as shown in Supplementary Fig. 15. The calculated $m^{*}$ (shown in Supplementary Fig. 16) along $X-K$ direction is $\sim 10$ times larger than that along $\Gamma-X$, which seems to lead to a better transport property along $x$ axis than $y$ axis. However, deformation potential $E_{1}$ (shown in Supplementary Fig. 16) due to electron-phonon scattering shows an opposite trend to $m^{*}$ in both $x$ and $y$ axes, and dominates over the anisotropy of effective mass to give rise to the anisotropy of electrical conductivity $\sigma$ according to the deformation potential theory (see Methods), $\sigma_{\mathrm{y}}$ is about five times as big as $\sigma_{\mathrm{x}}$ at low doping. When plotting experimental data in the renormalized conductivity polar figure in Fig. 1j, a twofold symmetry of conductance can be clearly seen.

Gate-tunable GAR in ultra-thin GaTe. The above-mentioned twofold in-plane symmetry of resistivity can be reproduced in multiple GaTe devices (Supplementary Figs. 17-18), and we performed a systematic study on the sample S2. As shown in Fig. 2a, with the same GaTe flake (optical image of the device is shown in the inset of Fig. 2a), field-effect curves, measured at source-drain voltage $V_{\mathrm{ds}}=2 \mathrm{~V}$ along $x$ and $y$ directions, exhibit strong anisotropy, indicated by red and blue colors, respectively. $I_{\mathrm{ds}}$ measured along the 12 directions with a fixed $V_{\mathrm{ds}}=5 \mathrm{~V}$ are plotted at $V_{\mathrm{g}}=-60 \mathrm{~V}$, while an ellipsoidal oscillation at all angles with $2 \pi$ periodicity can be seen, shown in Fig. 2b. When the gate voltages were swept from $-60 \mathrm{~V}$ to $-30 \mathrm{~V}$, the maximum-to-minimum $I_{\mathrm{ds}}$ ratio of the ellipsoidal oscillation at each gate voltage varies largely. Note that similar gate-tunable anisotropic resistance behavior is also seen in bare GaTe devices without BN encapsulation (Supplementary Figs. 19-20).

Taking $y\left(0^{\circ}\right)$ and $x\left(90^{\circ}\right)$ directions for examples, closer look of $I-V$ output curves in the range of $\pm 5 \mathrm{~V}$ is shown in Fig. $2 \mathrm{c}-\mathrm{d}$. It is seen that at high bias voltage, $I_{\mathrm{ds}}$ for direction $y$ is varying within $10^{-7}$ and $10^{-5} \mathrm{~A}$, whereas $I_{\mathrm{ds}}$ for direction $x$ is varying within $10^{-11}$ and $10^{-6} \mathrm{~A}$. We define the electrical maximum anisotropic ratio $\Gamma_{\mathrm{a}}$ as $I_{\mathrm{y}} / I_{\mathrm{x}}$ for each fixed gate voltage. Strikingly, as extracted from Fig. $2 \mathrm{~b}, \Gamma_{\mathrm{a}}$ as a function of gate voltage shows a gate-tunability from less than one order to as large as $10^{3}$. This gate-tunable GAR effect was not found in previous studies. We fabricated various samples in similar configuration of h-BN encapsulated ultra-thin GaTe devices (Supplementary Figs. 21-23), with their $\Gamma_{\mathrm{a}}$ plotted together in Fig. 2e. In general, anisotropic ratio in those devices can be gate tuned from a few times, to several hundreds or even thousands folds, which is much higher than other $2 \mathrm{D}$ systems 

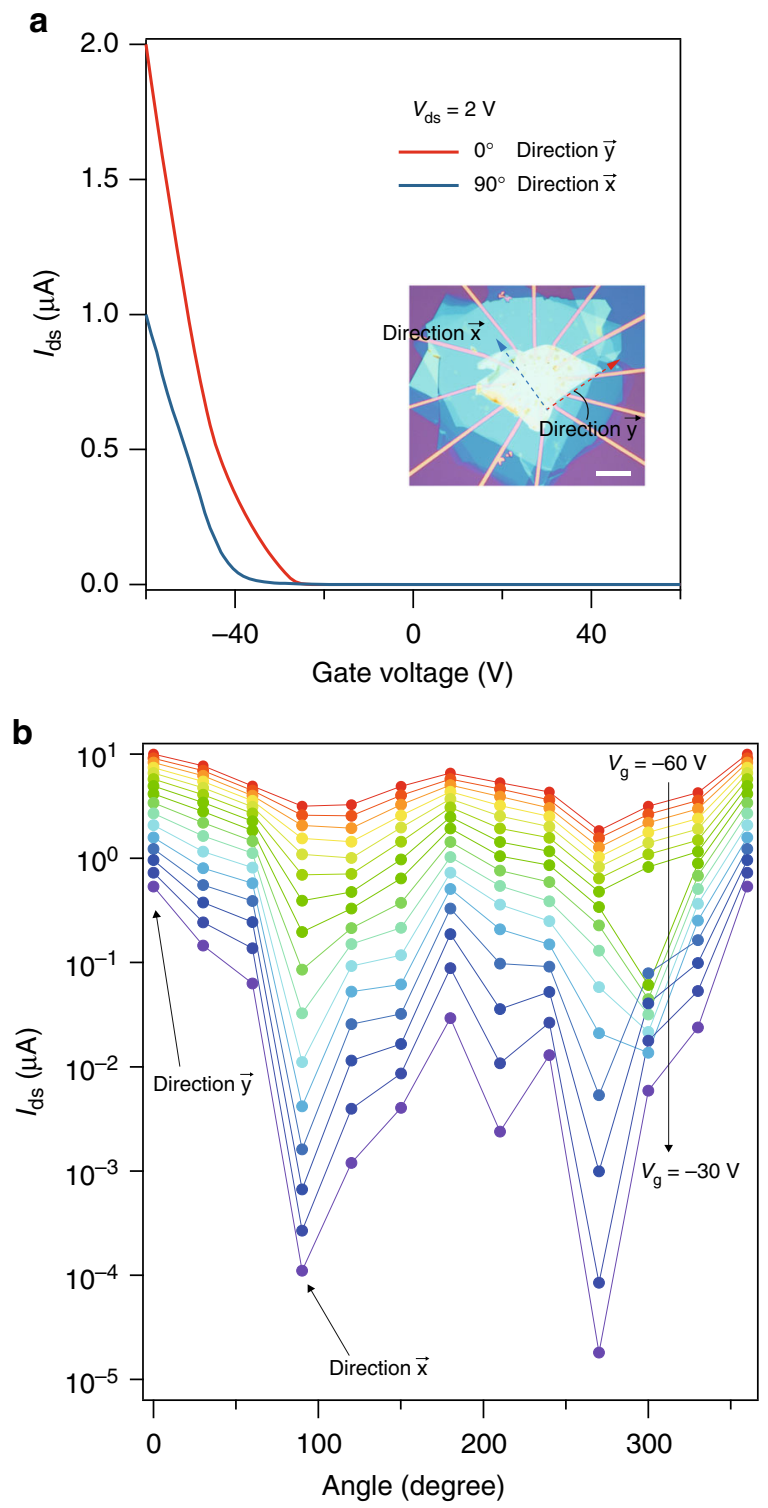
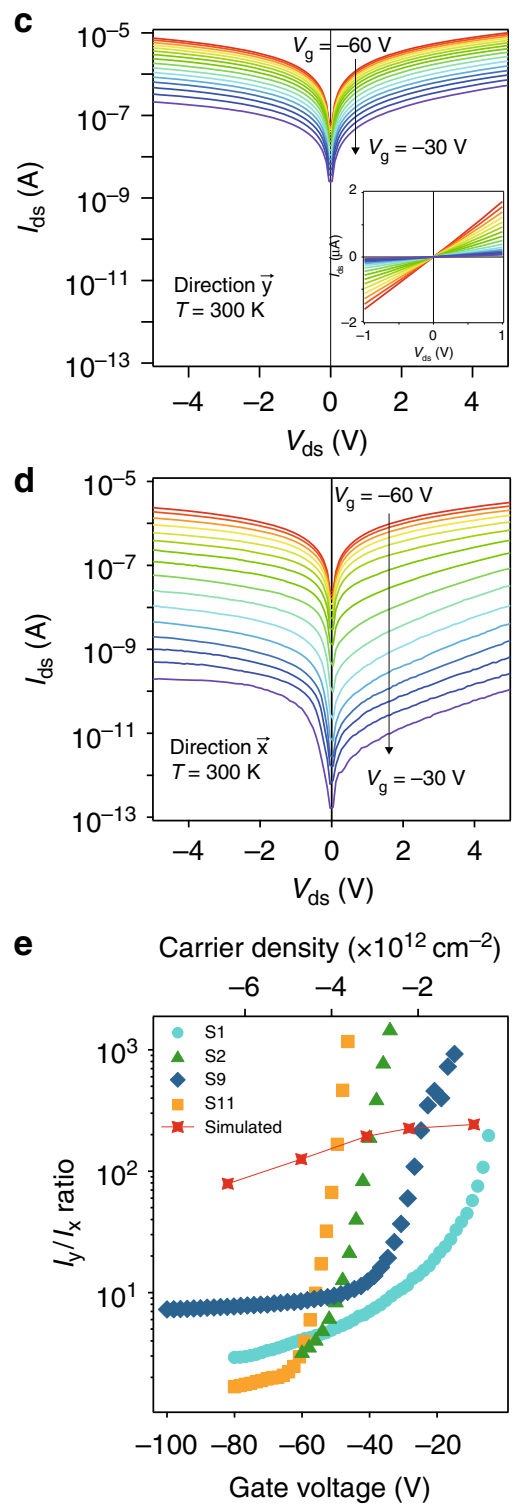

Fig. 2 Giant anisotropic resistance in ultra-thin GaTe. a Field effect curves of a typical GaTe device (Sample S2) recorded along $x$ and $y$ directions, with the optical image of the device shown in the inset. Scale bar in the inset is $10 \mu \mathrm{m}$. $\mathbf{b} I_{\mathrm{ds}}$ of the same device measured along the 12 directions with a fixed $V_{\mathrm{ds}}=5$ $\mathrm{V}$, and with the gate voltages swept from $-60 \mathrm{~V}$ to $-30 \mathrm{~V}$. c, d $I-\mathrm{V}$ curves plotted in log scale at $V_{\mathrm{ds}}= \pm 5 \mathrm{~V}$ of the same device measured along $y$ and $x$ directions, respectively. The gate voltages were swept from $-60 \mathrm{~V}$ to $-30 \mathrm{~V}$. Inset of $\mathbf{c}$ shows the corresponding data in linear scale. e The maximal electrical anisotropic ratio $I_{\mathrm{y}} / I_{\mathrm{x}}$ extracted from different samples (denoted as S1, S2, S9, and S11) as a function of gate voltage

reported so far $4,5,7,9,22$. To push the $2 \mathrm{D}$ limit of the GaTe thicknesses studied in this work, we managed to fabricate sample of $\mathrm{GaTe}$ with $\sim 4.8 \mathrm{~nm}$ in thickness. And as the inter-layer distance of GaTe is $\sim 0.8 \mathrm{~nm}^{21}$, we can draw the conclusion that, down to the six-layer limit, the observed GAR prevails, as shown in Supplementary Fig. 24.

As $\Gamma_{\mathrm{a}}$ in ultra-thin GaTe is rather weak at relatively higher hole doping (say, in the $V_{\mathrm{g}}=-60 \mathrm{~V}$ limit), and becomes significantly enhanced close to the VBM in the vicinity of $V_{\mathrm{g}}=-30 \mathrm{~V}$, it more or less shows a better electron collimation at higher $\Gamma_{\mathrm{a}}$ values, i.e., electrons tend to flow preferably along a certain direction at those conditions. Considering the deformation potential theory only applied to the band edge (VBM or CBM) and its inability to take care the gate-tunability on electronic transport ${ }^{23,24}$, we calculate $I-V$ curves and their gate dependence by combining density functional theory (DFT) calculation with the nonequilibrium Green's function (NEGF) method $^{25}$ (see Methods), and show calculated field effect I-V data at $V_{\mathrm{ds}}=0.5 \mathrm{~V}$ along two perpendicular crystalline directions in Supplementary Fig. 25. To avoid possible contact Schottky barrier, we use $p$-type heavily doped GaTe as source and drain. $I-V$ curves along both $x$ and $y$ directions show a similar behavior to the experimental ones as given in Fig. 2a. $I_{\mathrm{y}, \mathrm{ds}}$ at $V_{\mathrm{ds}}=0.5 \mathrm{~V}$ increases from $1.39 \mu \mathrm{A}$ at $V_{\mathrm{g}}=-9.1 \mathrm{~V}$ to $12.52 \mu \mathrm{A}$ at $V_{\mathrm{g}}=-82 \mathrm{~V}$. More strikingly is the gate dependence of $I_{\mathrm{x}, \mathrm{ds}}$, which changes by two orders of magnitude, from $4.63 \times 10^{-3} \mu \mathrm{A}$ at $V_{\mathrm{g}}=-9.1 \mathrm{~V}$ to $0.19 \mu \mathrm{A}$ at $V_{\mathrm{g}}=-82 \mathrm{~V}$. The calculated $I_{\mathrm{y}} / I_{\mathrm{x}}$ ratio, given in filled red squares in Fig. $2 \mathrm{e}$, decreases with increasing gate voltage, from $\approx 300$ at $-9.1 \mathrm{~V}$ to $\approx 60$ at $-82 \mathrm{~V}$, agreeing qualitatively with the experimental ratio but in a milder way than the experimental data. The discrepancy can be ascribed to that extrinsic effect in experiment such as nonlinear contact resistivity is not taken into account in the simulated device. Nevertheless, the gate-tunable GAR can still be intrinsically analyzed from the calculated 
transmission coefficient, as shown in Supplementary Fig. 26. At low gate voltage $(-9.1 \mathrm{~V})$, there is almost no $x$ direction transmission channels in the scattering region between source energy level $E_{\mathrm{L}}$ and drain energy level $E_{\mathrm{R}}$, whereas a sizeable $y$ direction transmission is observed, giving rise to a large anisotropic ratio $\Gamma_{\mathrm{a}}$ at low gate voltages. In contrast, at high gate voltage $(-82 \mathrm{~V})$, non-zero transmission coefficient in channel material appears with comparable total transmission in both $x$ and $y$ directions, greatly suppressing GAR in GaTe.

Ultra-thin GaTe anisotropic floating gate memory. It is of great importance to demonstrate conceptual devices taking advantage of the GAR effect in ultra-thin GaTe. In the following, we will discuss a prototype anisotropic memory based on ultra-thin GaTe. Using the vdW assembly method (see Methods), a few-layered graphene was equipped in addition to the h-BN/ GaTe/h-BN sandwich, forming an architecture of FGM. Optical image and art view of such typical devices are shown in Fig. 3a, b.
Interestingly, when sweeping within the same gate window, the hysteresis memory curves along $y$ and $x$ directions vary largely, with a clear discrepancy of memory window size, shown in Fig. $3 c$, d. This result is more pronounced in a log scale when plotted together in Fig. 3e. One can see that after an operation of erasing (sweep the $V_{\mathrm{g}}$ from $0 \mathrm{~V}$ to $-20 \mathrm{~V}$, and back to $0 \mathrm{~V}$ ), the device stays in an "Off" state in both directions. While an program operation (sweep the $V_{\mathrm{g}}$ from $0 \mathrm{~V}$ to $20 \mathrm{~V}$, and back to $0 \mathrm{~V}$ ), the device stays in two different "On" states in $y$ and $x$ directions, as indicated by colored circles in Fig. 3e, and their corresponding retention in Fig. 3f. It thus makes the device an anisotropic memory, i.e., one single operation of programmingerasing will generate two sets of data in the two directions, making the device conceptually compatible with directionsensitive data storage.

\section{Discussion}

To verify the performance of ultra-thin GaTe FGM with graphite floating gate along fixed direction, we recorded polarization a

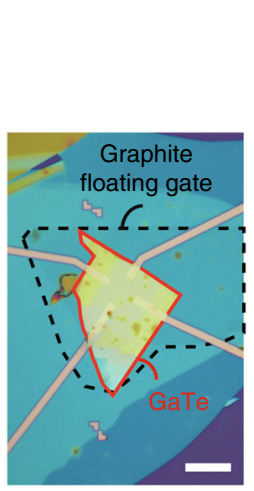

b

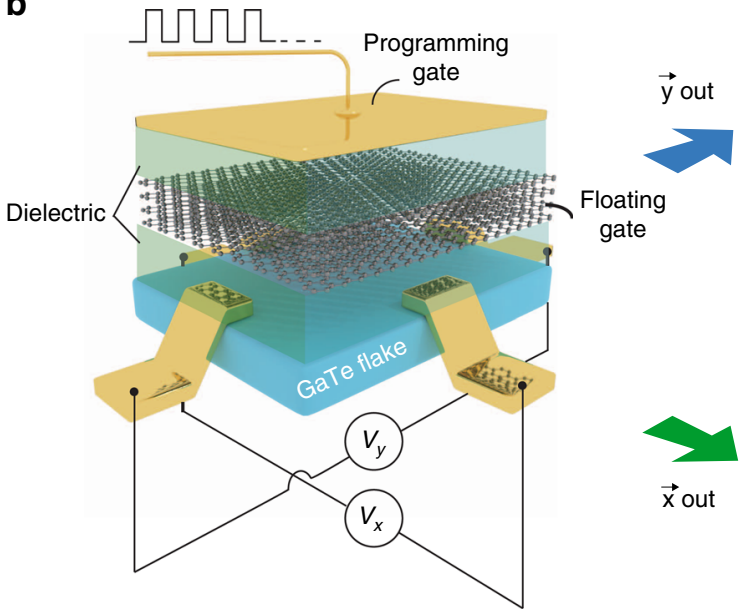

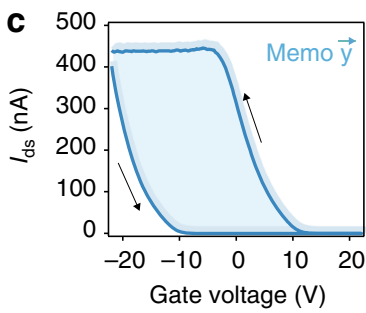

d

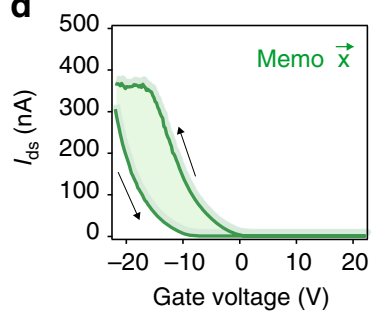

e

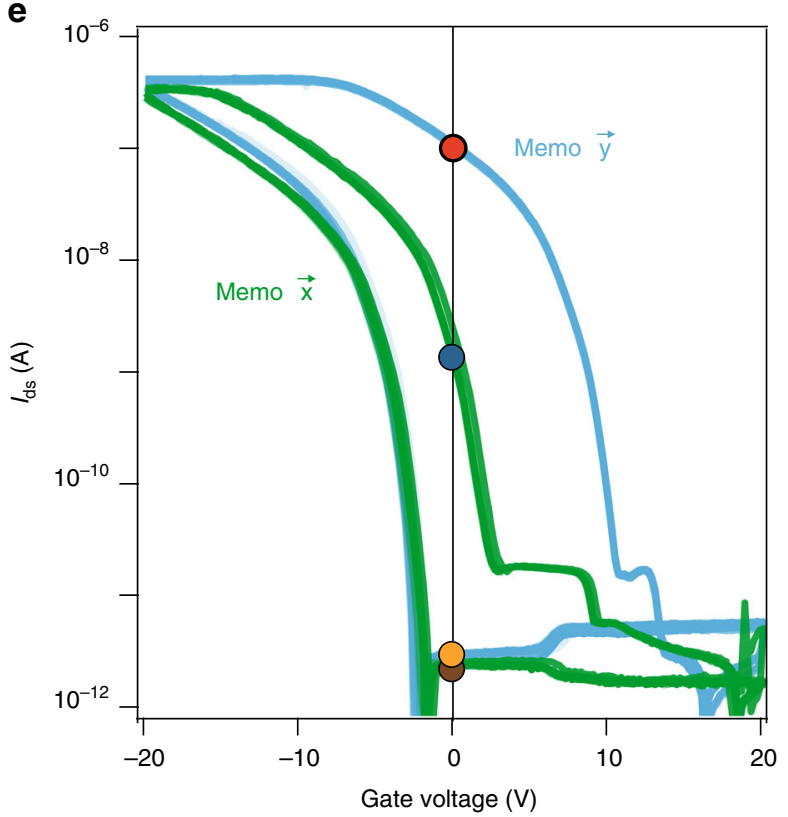

f

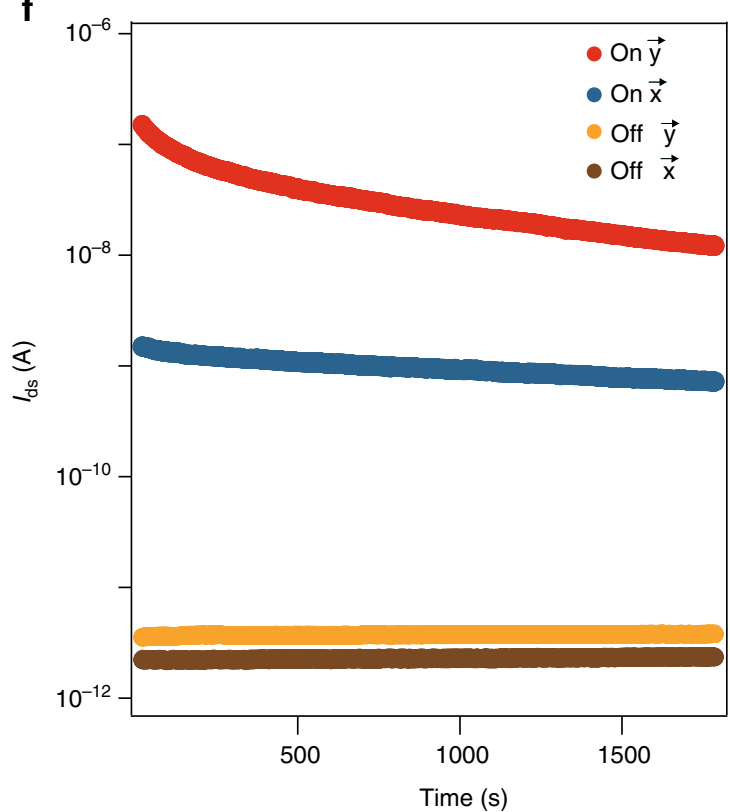

Fig. 3 Anisotropic ultra-thin GaTe floating gate memory. a, b Optical image and art view of a typical h-BN/GaTe/h-BN device (Sample S6) with a graphite floating gate. Scale bar in a is $10 \mu \mathrm{m}$. c, d Memory curves measured along $x$ and $y$ directions, respectively. e Same data in c and d, plotted in log scales, with 10 repeated measurements (indicated by changing the gradient of the green and blue colors). $\mathbf{f}$ Retention time of memory at $V_{g}=0 \mathrm{~V}$, along $y$ and $x$ directions, with on and off positions indicated by the colored circles in $\mathbf{e} . V_{\mathrm{ds}}=2 \mathrm{~V}$ was used in the above measurements 

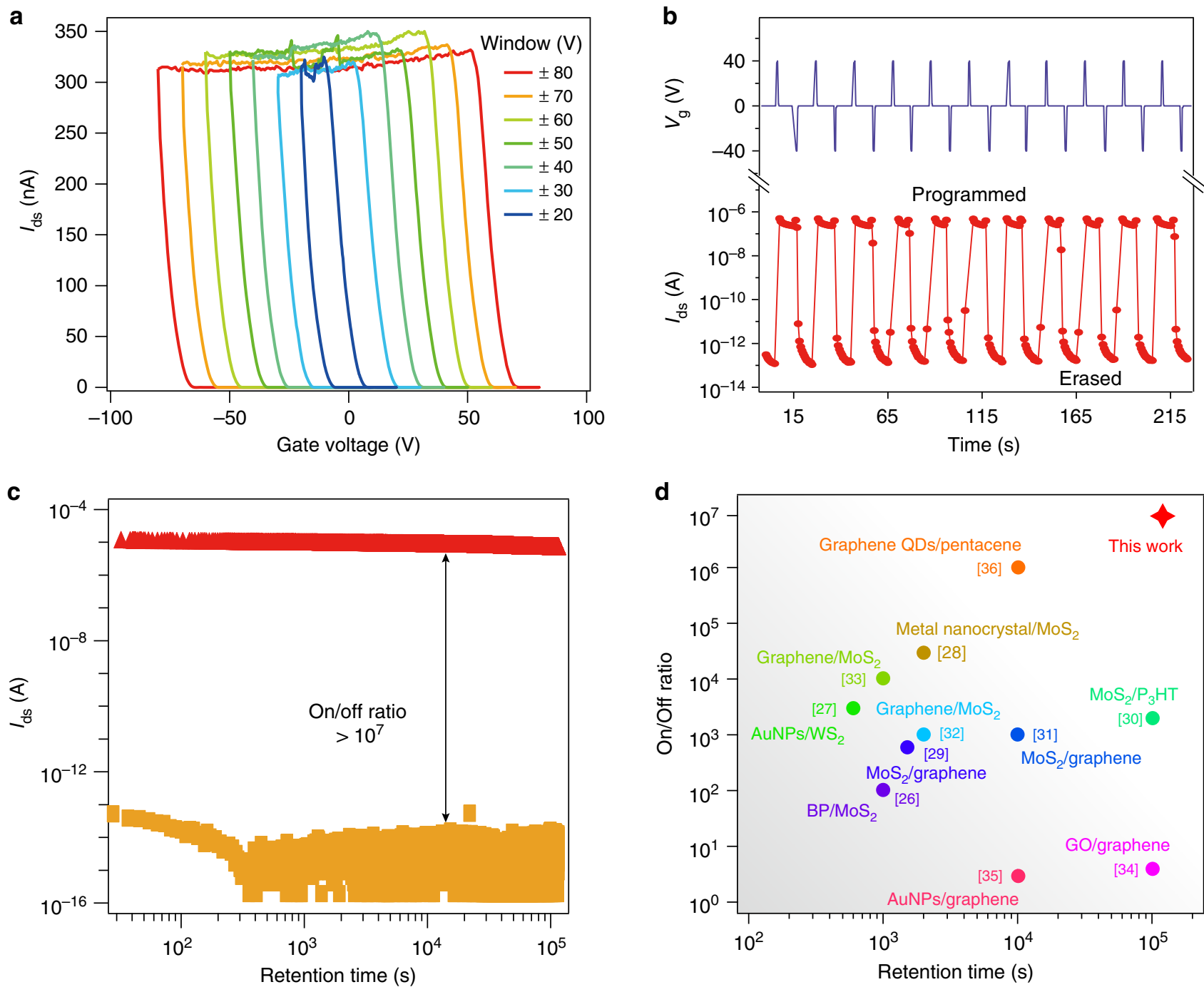

Fig. 4 Comparison of the floating gate memory performance of GaTe with other 2D materials. a Memory windows measured in y direction. $\mathbf{b}$ Demonstration of erasing and programming pulses measured in $y$ direction. c Retention time test of the device at on and off states. $\mathbf{d}$ Summary of the performance of FGM made by $2 \mathrm{D}$ materials. Data in $\mathbf{a}, \mathbf{b}$ are measured in sample $\mathrm{S} 6$, and data in $\mathbf{c}$, $\mathbf{d}$ are measured in sample $\mathrm{S} 4$. $V_{\mathrm{ds}}=2 \mathrm{~V}$ was used in the above measurements

gate voltages for different ranges from $\pm 20 \mathrm{~V}$ to $\pm 80 \mathrm{~V}$ in direction $y$, shown in Fig. 4 a (see also Supplementary Figs. 27-28). It can be seen that the GaTe memory shows rather stable erased state in all gate ranges. Once fed with the stimulation of pulsed $V_{\mathrm{g}}$ of $\pm 40 \mathrm{~V}$, the device can be readily erased and programmed as illustrated in Fig. $4 \mathrm{~b}$.

In the following, we compare the GaTe FGM with the state-ofthe-art FGMs fabricated from other 2D materials ${ }^{26-36}$. Given that retention of the erased and/or programmed states is one of the most important parameter of such FGM, we recorded along direction $y$ the on state (by a positive gate pulse), and the off state (by a negative gate pulse), respectively. Among GaTe FGM devices tested, the best performance (measured from sample S4 as shown in Fig. 4c) exhibits an on/off ratio exceeding $10^{7}$ and the attenuation of the on/off ratio is $<5 \%$ in a retention time of $10^{5} \mathrm{~s}$ (detailed memory characterizations of sample S4 are shown in Supplementary Fig. 29). By summarizing the state-ofthe-art performance of FGM based on 2D materials, it is found that, our h-BN/GaTe/h-BN with graphite floating gate memory shows up to now the record on/off ratio and retention time, as shown in Fig. 4d. We attribute the observed high on/off ratio in the h-BN encapsulated devices to the better preserved band gap in the pristine GaTe. When the Fermi level is tuned to be inside the band gap (no electron density of state available), the offstate current is at the order of $\mathrm{pA}$, whereas the on-state current reaches a few $\mu \mathrm{A}$. To illustrate the working principle of the GaTe floating gate memory devices, we show, according to previous studies $26,27,29,33$, simplified band diagram in Supplementary Fig. 30.

In conclusion, we have discovered that ultra-thin GaTe encapsulated by h-BN devices show a twofold symmetry electrical conductance oscillations along different in-plane directions, with their maximum anisotropic ratios $I_{\mathrm{y}} / I_{\mathrm{x}}$ gate-tunable in the range from $<10$ to a few thousands. This GAR arises from the in-plane anisotropic transmission coefficients, and the anisotropic ratio can be changed by several orders of magnitude mainly owing to the sensitivity of $x$ direction conduction channel to gating. Based on this GAR effect, we devised an anisotropic GaTe memory with a vdW assembled graphite floating gate. The prototype memory devices show direction-sensitive multiple-level output nonvolatile memory behavior when measured from different directions. Moreover, fixed direction measurements suggest that the GaTe FGMs show record performances in terms of both on/off ratio and retention time among the state-of-the-art FGM based 
on 2D materials. Using this unit cell of GaTe anisotropic field effect transistor as a building block, it can be further expanded into many possible applications such as neural computation with GaTe FGM neural network arrays.

\section{Methods}

Single crystal GaTe was prepared via the self-flux method. Raw bulk materials with stoichiometric ratio of $\mathrm{Ga}$ (purity 99.999\%): Te (purity 99.9\%) $=48.67: 51.33$ were mixed and kept at $880^{\circ} \mathrm{C}$ for $5 \mathrm{~h}$. The mixture was then cooled at the rate of $1.5^{\circ} \mathrm{C}$ $\mathrm{h}^{-1}$, followed by a natural cooling process. The h-BN (crystals from HQ Graphene) encapsulated GaTe devices were fabricated using the dry-transfer methods (Supplementary Fig. 31$)^{37}$, and then annealed in $\mathrm{Ar}: \mathrm{H}_{2}(10: 1)$ mixture at $320^{\circ} \mathrm{C}$ for 30 mins before electrical measurements.

The h-BN-encapsulated GaTe heterostructures were fabricated in an mBraununiverse glove box. A Bruker Dimension Icon AFM was used for thicknesses and morphology tests. Raman measurements were performed by an HR 800 JobinYvon Horiba polarized Raman spectroscopy. The electrical performances of the devices were measured using a semiconductor analyzer (Agilent B1500A) and a probe station (Cascade Microtech Inc. EPS150) under ambient conditions.

The electronic band structure in this work was calculated by using the firstprinciples density functional theory as implemented in the VASP code $^{38}$. Projector augmented wave pseudopotentials $s^{39}$ and the Perdew-Burke-Ernzerhof ${ }^{40}$ functional were, respectively, used to describe electron-ion interaction and electronic exchange-correlation interaction. We adopted $500 \mathrm{eV}$ as the electronic kinetic energy cutoff for the plane-wave basis and $10^{-6} \mathrm{eV}$ as the criterion for reaching self-consistency. The Brillouin zone (BZ) of the primitive unit cell (12 atoms per cell) was sampled by $20 \times 20 \times 1$ k-points ${ }^{41}$. Rectangle supercell ( 24 atoms per cell) was used to calculate anisotropic electrical conductivity and the BZ was sampled by $2 \times 6 \times 1$ k-points.

The electrical conductivity $\sigma$ was evaluated based on $\sigma=$ ne $\mu$, in which carrier mobility $\mu$ was calculated based upon the deformation potential theory with formula ${ }^{23,24}$

$$
\mu=\frac{e \hbar^{3} C_{2 D}}{k_{B} T m^{*} m_{d} E_{1}^{2}},
$$

where $\hbar$ is the reduced Planck constant, $C_{2 \mathrm{D}}$ is the elastic modulus derived from ( $E$ $\left.-E_{0}\right) / A_{0}=C \varepsilon^{2} / 2\left(E, E_{0}, A_{0}, C\right.$, and $\varepsilon$ denote, respectively, the total energy, the total energy at equilibrium, the area of the $2 \mathrm{D}$ unit cell at equilibrium, elastic constant, and the lattice strain), $k_{\mathrm{B}}$ is the Boltzmann constant, $T$ is temperature, $m^{*}$ is the effective mass in the transport direction, $m_{\mathrm{d}}$ is the averaged effective mass defined by $m_{d}=\sqrt{m_{x}^{*} m_{y}^{*}}$ and $E_{1}$ is the deformation potential constant of the VBM along the transport direction. $E_{1}$ defined by $E_{1}=\Delta V / \varepsilon$ with $\Delta V$ as the energy change of the VBM under small strain $\varepsilon$.

$I-V$ curve of the GaTe transistor was simulated using the DFT coupled with the NEGF method ${ }^{25}$, as implemented in the ATK package ${ }^{42}$. The structure of GaTe transistor is shown in Supplementary Fig. 25(a). We employed the Dirichlet boundary condition to ensure the charge neutrality in the source and the drain region. The temperature was set to $300 \mathrm{~K}$. The density mesh cutoff was set to 80 Hartrees. The Monkhorst-Pack k-point meshes for device along the $x$ and $y$ direction were sampled with $40 \times 6 \times 1$ and $114 \times 6 \times 1$, respectively. Electron-ion interaction was treated by SG15 Optimized Norm-Conserving Vanderbilt (ONCV) pseudopotentials ${ }^{43,44}$. The transmission coefficient at energy $\mathrm{E}$ was averaged over $31 \mathrm{k}$-points perpendicular to the transport direction. The generalized gradient approximation was adopted for the electronic exchange-correction functional. The length of scattering region is $9.530 \mathrm{~nm}$ and $9.676 \mathrm{~nm}$ for transistor along $x$ and $y$ directions, respectively, both of which are sufficiently large to avoid interaction between source and drain. The code of VASP (www.vasp.at) and QuantumATK (https://docs.quantumwise.com/guides/guides.html) are commercially available from their official websites.

\section{Data availability}

The data that support the findings of this study are available from the corresponding authors upon reasonable request.

Received: 18 April 2019 Accepted: 2 May 2019

Published online: 24 May 2019

\section{References}

1. Krishnan, K. S. \& Ganguli, N. Large anisotropy of the electrical conductivity of graphite. Nature 144, 667 (1939).

2. Eltsev, Y. et al. Anisotropic resistivity and hall effect in $\mathrm{MgB}_{2}$ single crystals. Phys. Rev. B 66, 180504(R) (2002).

3. Zhao, Y. et al. Anisotropic magnetictransport and exotic longitudinal linear magnetoresistance in $\mathrm{WTe}_{2}$ crystals. Phys. Rev. B 92, 041104(R) (2015).
4. Xia, F., Wang, H. \& Jia, Y. Rediscovering black phosphorus as an anisotropic layered material for optoelectronics and electronics. Nat. Commun. 5, 4458 (2014).

5. Chen, Y. et al. Black arsenic: a layered semiconductor with extreme in-plane anisotropy. Adv. Mater. 30, 1800754 (2018).

6. $\mathrm{Xu}, \mathrm{X}$. et al. In-plane anisotropies of polarized raman response and electrical conductivity in layered tin selenide. ACS Appl. Mater. Interfaces $\mathbf{9}$, 12601-12607 (2017).

7. Liu, Y. et al. Raman signatures of broken inversion symmetry and in-plane anisotropy in type-II Weyl semmimetal candidate TaIrTe 4 . Adv. Mater. 30, 1706402 (2018).

8. $\mathrm{Li}, \mathrm{L}$. et al. Strong in-plane anisotropies of optical and electrical response in layered dimetal chalcogenide. ACS Nano 11, 10264-10272 (2017).

9. Liu, E. et al. Integrated digital inverters based on two-dimensional anisotropic $\mathrm{ReS}_{2}$ field-effect transistors. Nat. Commun. 6, 6991 (2015).

10. Li, L. et al. 2D GeP: An unexploited low-symmetry semiconductor with strong in-plane anisotropy. Adv. Mater. 30, 1706771 (2018).

11. Yang, S. et al. Highly in-plane optical and electrical anisotropy of 2D germanium arsenide. Adv. Funct. Mater. 28, 1707379 (2018).

12. Huang, S. et al. In-plane optical anisotropy of layered gallium telluride. ACS Nano 10, 8964-8972 (2016).

13. Sánchez-Royo, J. F. et al. Angle-resolved photoemission study and firstprinciples calculation of the electronic structure of GaTe. Phys. Rev. B 65, 115201 (2002)

14. Tatsuyama, C., Watanabe, Y., Hamaguchi, C. \& Nakai, J. Some optical properties of layer-type semiconductor GaTe. J. Phys. Soc. Jpn. 29, 150 (1970).

15. Liu, F. et al. High-sensitivity photodetectors based on multilayer GaTe flakes. ACS Nano 8, 752-760 (2014).

16. Leão, C. R. \& Lordi, V. Ab initio guided optimization of GaTe for radiation detection applications. Phys. Rev. B 84, 165206 (2011).

17. Rahmlow, T. D. Jr. et al. Development of front surface, spectral control filters with greater temperature stability for thermophotovoltaic energy conversion. AIP Conf. Proc. 890, 59 (2007).

18. Fonseca, J. J. et al. Bandgap restructuring of the layered semiconductor gallium telluride in air. Adv. Mater. 28, 6465-6470 (2016).

19. $\mathrm{Hu}, \mathrm{P}$. et al. Highly sensitive phototransistors based on two-dimensional GaTe nanosheets with direct bandgap. Nano Res. 7, 694-703 (2014).

20. Yang, S. X. et al. Highly-anisotropic optical and electrical properties in layered SnSe. Nano. Res. 11, 554-564 (2018).

21. Wang, Z. et al. Role of Ga vancancy on a multilayer GaTe phototransistor. ACS Nano 8, 4859-4865 (2014).

22. Qiu, G. et al. Observation of optical and electrical in-plane anisotropy in highmobility few-layer $\mathrm{ZrTe}_{5}$. Nano. Lett. 16, 7364-7369 (2016).

23. Bardeen, J. \& Shockley, W. Deformation potentials and mobilities in nonpolar crystals. Phys. Rev. 80, 72-80 (1950).

24. Dong, B. et al. New two-dimensional phase of tin chalcogenides: candidates for high-performance thermoelectric materials. Phys. Rev. Mater. 3, 013405 (2019).

25. Brandbyge, M., Mozos, J.-L., Ordejón, P., Taylor, J. \& Stokbro, K. Densityfunctional method for nonequilibrium electron transport. Phys. Rev. B 65, 165401 (2002).

26. Li, D. et al. Nonvolatile floating-gate memories based on stacked black phosphorus-boron nitride- $\mathrm{MoS}_{2}$ heterostructures. Adv. Funct. Mater. 25, 7360-7365 (2015).

27. Gong, F. et al. High-sensitivity floating-gate phototransistors based on $\mathrm{WS}_{2}$ and $\mathrm{MoS}_{2}$. Adv. Funct. Mater. 26, 6084-6090 (2016).

28. Wang, J. et al. Floating gate memory-based monolayer $\mathrm{MoS}_{2}$ transistor with metal nanocrystals embedded in the gate dielectrics. Samll 11, 208-213 (2015).

29. Choi, M. S. et al. Controlled charge trapping by molybdenum disulphide and graphene in ultrathin heterostructured memory devices. Nat. Commun. $\mathbf{4}$, 1624 (2013).

30. Kang, M. et al. Stable charge storing in two-dimensional $\mathrm{MoS}_{2}$ nanoflake floating gates for multilevel organic flash memory. Nanoscale 6, 12315 (2014).

31. Vu, Q. A. et al. Two-terminal floating-gate memory with van der waals heterostructures for ultrahigh on/off ratio. Nat. Commun. 7, 12725 (2016).

32. $\mathrm{Vu}, \mathrm{Q}$. A. et al. A high-on/off-ratio floating-gate memristor array on a flexible substrate via CVD-grown large-area 2D layer stacking. Adv. Mater. 29, 1703363 (2017).

33. Bertolazzi, S., Krasnozhon, D. \& Kis, A. Nonvolatile memory cells based on $\mathrm{MoS}_{2}$ /graphene heterostructures. ACS Nano 7, 3246-3252 (2013).

34. Jang, S., Hwang, E., Lee, J. H., Park, H. S. \& Cho, J. H. Graphenegraphene oxide floating gate transistor memory. Small 11, 311-318 (2015).

35. Jang, S., Hwang, E. \& Cho, J. H. Graphene nano-floating gate transistor memory on plastic. Nanoscale 6, 15286 (2014). 
36. Ji, Y. et al. Graphene quantum dots as a highly efficient solution-processed charge trapping medium for organic nano-floating gate memory. Nanotechnology 27, 145204 (2016).

37. Wang, Z. et al. Electric-field control of magnetism in a few-layered van der waals ferromagnetic semiconductor. Nat. Nanotechnol. 13, 554-559 (2018).

38. Kresse, G. \& Furthmüller, J. Efficient iterative schemes for $a b$ initio total-energy calculations using a plane-wave basis set. Phys. Rev. B 54, 11169-11186 (1996).

39. Kresse, G. \& Joubert, D. From ultrasoft pseudopotentials to the projector augmented-wave method. Phys. Rev. B 59, 1758-1775 (1999).

40. Perdew, J. P., Burke, K. \& Ernzerhof, M. Generalized gradient approximation made simple. Phys. Rev. Lett. 77, 3865-3868 (1996).

41. Monkhorst, H. J. \& Pack, J. D. Special points for brillouin-zone integrations. Phys. Rev. B 13, 5188-5192 (1976).

42. QuantumATK version 2018.06. Synopsys QuantumWise A/S, https://www. synopsys.com/silicon/quantumatk.html.

43. Hamann, D. R. Optimized norm-conserving vanderbilt pseudopotentials. Phys. Rev. B 88, 085117 (2013).

44. Schlipf, M. \& Gygi, F. Optimization algorithm for the generation of oncv pseudopotentials. Comput. Phys. Commun. 196, 36-44 (2015).

\section{Acknowledgements}

This work is supported by the National Key R\&D Program of China (2017YFA0206302), and is supported by the National Natural Science Foundation of China (NSFC) with Grant 11504385 and 51627801. T. Yang acknowledges supports from the Major Program of Aerospace Advanced Manufacturing Technology Research Foundation NSFC and CASC, China (no. U1537204). L.T. Xiao acknowledges support from the National Key R\&D Program of China (2017YFA0304203). H.H. Guo acknowledges NSFC Grant no. 51702146. Z. Han acknowledges the support from the Program of State Key Laboratory of Quantum Optics and Quantum Optics Devices (no. KF201816).

\section{Author contributions}

Z.H., Z.Z. and T.Y. conceived the experiment and supervised the overall project. H.W., X.S., J.C. fabricated the devices. H.W., M.C., X.S., X.L., Y.Y. and Z.H. carried out electronic transport measurements; M.Z., C.Q., X.Z., L.X., J.Z., S.C. and J.C. carried out optical measurements; Y.W., B.D. and T.Y. conducted the theoretical simulations. J.H. and H.G. did the simulations on polarized Raman. D.S. contributed in sample fabrications. L.Z. and W.L. grew the GaTe bulk crystals. K.S., J.W. and Y.H. carried out TEM performances. The manuscript was written by Z.H., T.Y. and H.W. with discussion and inputs from all authors.

\section{Additional information}

Supplementary Information accompanies this paper at https://doi.org/10.1038/s41467019-10256-3.

Competing interests: The authors declare no competing interests.

Reprints and permission information is available online at http://npg.nature.com/ reprintsandpermissions/

Journal peer review information: Nature Communications thanks the anonymous reviewer(s) for their contribution to the peer review of this work. Peer reviewer reports are available.

Publisher's note: Springer Nature remains neutral with regard to jurisdictional claims in published maps and institutional affiliations.

(c) (i) Open Access This article is licensed under a Creative Commons Attribution 4.0 International License, which permits use, sharing, adaptation, distribution and reproduction in any medium or format, as long as you give appropriate credit to the original author(s) and the source, provide a link to the Creative Commons license, and indicate if changes were made. The images or other third party material in this article are included in the article's Creative Commons license, unless indicated otherwise in a credit line to the material. If material is not included in the article's Creative Commons license and your intended use is not permitted by statutory regulation or exceeds the permitted use, you will need to obtain permission directly from the copyright holder. To view a copy of this license, visit http://creativecommons.org/ licenses/by/4.0/.

(C) The Author(s) 2019 